\section{Narrar la experiencia: procesos de registro en las prácticas de extensión universitaria}

\author{
María del Valle Bácerez \\ mvbacerez@gmail.com \\ (iD) orcid.org/0000-0003-3020-5595

\section{Nanci Díaz} \\ diaznanci@gmail.com \\ (iD) orcid.org/0000-0002-7022-2229 \\ Universidad Nacional del Litoral, Argentina
}

Sujetos y relaciones en extensión universitaria / Perspectivas

\section{다(1)(2)}

RECEPCIÓN: 26/06/20

ACEPTACIÓN FINAL: 15/09/20

\section{Resumen}

En el presente artículo queremos compartir algunas reflexiones en torno a la importancia del registro como herramienta que consideramos valiosa para recuperar por escrito las diferentes experiencias de las prácticas de extensión. Dicho análisis surge a partir de una serie de talleres de formación, convocadas por la Secretaría de Extensión Social y Cultural de la Universidad Nacional del Litoral, donde nos centramos precisamente en abordar los registros en el marco de los procesos de prácticas de extensión universitaria. A lo largo del escrito nos proponemos invitarles a reflexionar sobre el protagonismo que adquieren los estudiantes a partir de narrar sus propias experiencias de prácticas de extensión; los registros como materia prima para la construcción de conocimiento situado; y por último, el valor pedagógico de registro en el proceso de formación.

Palabras clave: registro; extensión universitaria; narrar experiencias.
Narrate the experience: registration processes in university extension practices

\section{Abstract}

This article aims to share reflections regarding the importance of data recording as a valuable tool to retrieve in writing the different experiences within university extension practices. Such analysis arises from a series of training workshops convened by the Social and Cultural Secretary of Extension of Universidad Nacional del Litoral, focus of our recorded data analysis -framed within university extension activities.

Throughout the article we attempt to make the reader reflect on the relevance acquired by: (i) students as narrators of their own experiences within university extension practices; (ii) recorded data as raw material for the elaboration of situated knowledge; and (iii) data recording -and its pedagogical value, as part of training processes.

Keywords: data recording; university extension; narrated experiences.
Narrar a experiência: processos de registro nas práticas de extensão universitária

\section{Resumo}

Neste artigo queremos compartilhar algumas reflexões sobre a importância do registro como ferramenta que consideramos valiosa para resgatar por escrito as diferentes experiências de práticas extensionistas. Esta análise surge a partir de uma série de oficinas de formação, convocadas pela Secretaria de Extensão Social e Cultural da Universidade Nacional del Litoral, onde nos concentramos justamente em abordar os registros no âmbito dos processos de práticas de Extensão Universitária.

Ao longo da redação propomos convidá-lo a refletir sobre o papel que os alunos adquirem ao narrar suas próprias experiências de práticas de extensão; os registros como matéria-prima para a construção do conhecimento situado; e, por fim, o valor pedagógico da inscrição no processo formativo.

Palavras-chave: registro; extensão universitária; narrar experiências.

Para citación de este artículo: Bácerez, M. del V. y Díaz, N. (2020). Narrar la experiencia: procesos de registro en las prácticas de extensión universitaria. +E: Revista de Extensión Universitaria, 10(13), e0013. doi: 10.14409/extension.2020.13.Jul-Dic.e0013 


\section{Introducción}

A partir de una serie de talleres de formación convocados por la Secretaría de Extensión Social y Cultural de la Universidad Nacional del Litoral (UNL), nos centramos en abordar los registros en el marco de los procesos de prácticas de extensión universitaria. Recuperando lo trabajado en dichos espacios formativos para la extensión y desde nuestra disciplina, Trabajo Social, el registro es una herramienta imprescindible, una técnica central para el desarrollo de estrategias de intervención pedagógico-sociales que contribuyen a una formación disciplinar al integrar teorías y prácticas propuestas en las cátedras y proyectos vigentes.

En este sentido, queremos compartir algunas reflexiones en torno a la importancia del registro como una herramienta valiosa escritural para recuperar las experiencias de los mismos participantes en el marco de dichas prácticas de extensión. Pero, antes de avanzar, consideramos pertinente comentar algunos aspectos respecto de las prácticas de extensión universitaria. En primer lugar, hace un tiempo ya que se viene debatiendo en cuanto a la institucionalidad de la extensión universitaria como parte de la vida académica, junto y al mismo nivel que la investigación y docencia. En segundo lugar, se requiere de la construcción del reconocimiento académico en la integración de tales prácticas de extensión en el currículo de formación que contemple las características propias que implican, diferentes de la investigación y de la docencia pero no incompatibles.

Y en tercer lugar, nos interesa resaltar la dimensión pedagógica de la extensión. No obstante, siguiendo a Menéndez (2011), podemos reconocer distintas dimensiones que constituyen las prácticas de extensión universitaria: por un lado, la dimensión académico-institucional refiere a esto que decíamos arriba, el reconocimiento de la extensión como una función sustantiva, integrada a la docencia y a la investigación; la dimensión social, cultural y productiva que, según el autor, "remite al rol que adquiere la extensión universitaria como acción que promueve una mejor calidad de vida, una mayor inclusión y cohesión social, un desarrollo humano y sustentable" (Menéndez, 2011, p. 24).

Por otra parte, la dimensión comunicacional alude al proceso de práctica o acción interventiva entendida desde el diálogo, las relaciones vinculares y la construcción mutua como colectiva. Se centra en sumar esfuerzos en la reflexión crítica y la valoración de cada sujeto con sus conocimientos y saberes propios que abonen a la transformación social. Por último, la dimensión educativa- pedagógica, que es la cual nos centraremos en este artículo, sin desentendernos del resto de las dimensiones ya que están íntimamente vinculadas ya que cada una abona a la configuración conceptual de la extensión universitaria entendida desde esta perspectiva.

Ahora bien, retomando la dimensión pedagógica, no desconocemos que existen diferentes posicionamientos metodológicos, epistemológicos y ético-políticos acerca de la extensión. No obstante, desde nuestro posicionamiento, coincidimos con Rafaghelli (2013):

"Entendemos la extensión universitaria como espacio de prácticas sociales compartidas y colaborativas para la construcción de propuestas alternativas. Estas actividades se consolidan mediante las acciones conjuntas que se tejen en los escenarios sociales/ culturales/históricos con el propósito de comprender e intervenir de manera creativa e innovadora en las situaciones identificadas como problemas sociales". (p. 23) 
A este respecto, como dijimos, compartiremos en este escrito algunas reflexiones que atañen a la importancia del registro como una valiosa herramienta escritural para recuperar dichas prácticas de educación experiencial y de extensión (Camilloni, 2013). Para eso proponemos algunos ejes de reflexión que orientan el relato: el protagonismo que adquieren los y las estudiantes a partir de narrar, construir relatos de las propias experiencias de prácticas de extensión, los registros como materia prima para la construcción de conocimiento situado, y el valor pedagógico del registro en el proceso de formación.

\section{Estar ahí... algunas reflexiones sobre el acontecer de los sujetos y del encuentro}

Partimos de las palabras de Jorge Larrosa, quien expresa que "la experiencia es eso que me pasa. No eso que pasa, sino eso "que me pasa'" (2009, p.14). Para este autor implica un modo de estar en el mundo, de habitar el mundo donde se suscita lo que identifica como "acontecimientos", dando a entender que pasa algo pero que ese algo no soy YO. Ese algo que acontece está fuera de mí, fuera del nosotros, no puedo controlarlo y ni siquiera comprenderlo, escapa a nuestro dominio, en una dinámica recursiva donde lo externo y nuestro mundo interno entran en tensión. Siguiendo a Argumedo (1993), consideramos que los acontecimientos siempre son políticos en tanto aparecen como una síntesis de innumerables factores: económicos, sociales, políticos, culturales, tecnológicos, organizativos, individuales, que se encuentran particularmente conjugados... En este sentido, lo que acontece nos provoca y desafía nuestra presencia de sujetos portadores de los atributos de una cultura, historia y de una versión de la modernidad, en el mundo de la vida cotidiana.

Entonces es interesante profundizar y analizar el estar ahí... de manera prolongada en el tiempo, en el marco de un proyecto de extensión. Desde nuestra perspectiva, la extensión propone encuentros en un escenario del mundo social abriendo la posibilidad de experiencias y vivencias que son una oportunidad cognoscitiva valiosa donde podemos:

"abordar las grandes preguntas sociales mediante estudios en pequeños mundos en los que es posible, como potenciales investigadores, observar y acercarnos personalmente a las vicisitudes de la vida cotidiana y a los significados que éstas tienen para los habitantes del lugar". (Rockwell, 2005, p. 3)

En este sentido, el acercamiento y participación en un espacio, las actividades, la cotidianeidad están atravesados por una relación social, objetivos prácticos y teóricos como horizontes de sentidos de tales prácticas (el para qué). Desde este lugar, una de las reflexiones que nos interesa compartir es pensar la estrategia de intervención de cada proyecto en términos etnográficos de "campo" que, siguiendo a Guber (2009), son una referencia empírica de análisis compuesta por la conjunción del ámbito físico, de les actores y actoras y de las actividades. Es un recorte de lo real que en este saber-hacer también podemos conocer para crear, visibilizar aspectos problemáticos que aparecen en la dinámica del entramado relacional que acontece. Asimismo, entendemos que la sola presencia en el campo no garantiza un acceso acabado

"y completo al objeto, en tanto no se reconozca la necesaria mediación teórica del sujeto cognoscente. Es desde el bagaje conceptual y de sentido común que se pueden aprehender realidades sociales, en el seno de una relación donde se van aprehendiendo recíprocamente dos mundos culturales". (Guber, 2001, p. 78) 
Ahora bien, el estar ahí nos desafía a la pregunta: ¿qué cosas nos suceden en el contacto con otros y otras? ¿Para qué estar ahí? En principio, podemos decir que el encuentro en el campo genera una tensión fundante e inevitable del estar ahí donde cada presencia contiene un bagaje cultural y en el intercambio, en el vínculo que gesta la experiencia misma, se van constituyendo tiempos, ritmos e intensidades singulares de tal dinámica. En la experiencia del trabajo de campo con mujeres de la comunidad Mocoví y el Consejo de Lengua y Cultura, la presencia -mujeres blancas criollas-implicó sentirnos observadas en el proceso de comunicación en las múltiples dimensiones de la palabra dicha o por decir, hasta en las miradas y los silencios. Aun luego de varios encuentros y meses de trabajo con ambos grupos, coincidimos en que los intercambios colectivos que se daban en las charlas grupales en las cuales nos expresamos desde la propia experiencia de "ser mujer", "miembro de un proyecto de extensión", "parte de la universidad: docente o estudiante", seguíamos sintiendo el peso de las miradas, los silencios invisibilizantes, el estado de atención vigilante. Esas miradas observadoras y esos silencios generaban mucha incomodidad, despertaban temores con relación a "no poder" comunicarnos. En los primeros intercambios o participación en las rondas, cuando hablábamos para compartir alguna vivencia propia u opinar respecto de la gestión del proyecto, se observaba en las miradas y gestos de las mujeres, pero más todavía en los hombres expresiones de desconcierto, de no entender lo que se estaba diciendo. Esto nos hizo prestar atención al propio lenguaje y reflexionar que las palabras provenían y tienen sentido según el contexto cultural y social en que se expresen. Al principio, las situaciones aludidas generaron impotencia pero al mismo tiempo implicaron parte del proceso de reflexividad del trabajo extensionista, que produjo transformación, incluso al comprender que nuestro mundo cultural y el del otro pueden no necesariamente encontrarse en todos los aspectos del proceso; que en el encuentro humano debemos entender que para que el otro no desaparezca el desencuentro es necesario y liberador.

De modo que pensar el estar ahí desde una actitud investigativa, indagatoria y de intervención, nos permite "aprehender el proceso social en su aspecto vivo por intermedio de nuestra condición de seres vivos" (Quirós, 2014, p. 51). Es decir, esto nos posibilita, por un lado, poder vivenciar, experimentar, pasar por el cuerpo aquel encuentro, el hecho de "dejarnos afectar" con todo lo que ello implica en tal relación, dejarnos interpelar en nuestros propios marcos conceptuales y culturales, la propia condición de mujer, la condición de clase, así como los prejuicios, los temores y las expectativas...

Recuperar ese estar ahí en un tiempo prolongado permite visibilizar esas experiencias, esos "dejarse afectar" de los momentos cotidianos compartidos, desde armar un mate con conversaciones de fondo hasta escribir un cartel de invitación a alguna actividad. Es ahí donde consideramos que se adquiere un valor cognoscente de gran importancia, ya que es en esos vínculos en los que se crean redes de "interconocimiento" (Quirós, 2014; Guber; 2004) donde los sujetos hacen, deshacen, transforman ese-su universo. Así, mediado por la teoría, podemos capturar no solo lo que hacen sino, y fundamentalmente, cómo lo hacen en el proceso vivido que nos habilita la experiencia de encuentro y participación en el universo de vínculos, como forma de conocer y de producir conocimiento. Siguiendo nuevamente a Quirós (2014): "interrogar ese cómo no es otra cosa que dar estatuto epistemológico a todo aquello que estamos en condiciones de captar y percibir, en virtud y por intermedio de nuestra convivencia con los otros" (p. 53). 
Por otra parte, la relación y el vínculo que generamos entre los y las participantes en lo que acontece en las prácticas de extensión no solo está atravesada por el diálogo, la palabra dicha, sino también, algo sumamente enriquecedor, por la experiencia de vincularnos a través del cuerpo, de las sensaciones, del silencio, de las miradas, de lo dicho como de lo no dicho, del afecto. De acuerdo con Marcio Goldman (2006), podemos decir que esta propuesta de aprendizaje requiere "poner el cuerpo", "hacer relaciones", donde la sociabilidad se convierte en objeto y medio de análisis, de reflexión. Y dejarse afectar (Favret-Saada; 1990; Quirós, 2014) implica una decisión, un posicionamiento metodológico, epistemológico y político.

Otro aspecto que queremos compartir se vincula con las posibilidades y limitaciones de acceso al universo que se desea conocer para hacer; aquí serán los mismos actores y actoras que pondrán los límites de participación en relación con los espacios y circuitos de intimidad social. Depende de esos modos de estar ahí que habilitarán o no diferentes espacios, actividades y circuitos que tengan que ver con la intimidad social o con instancias más formales. Esta cuestión es de mayor importancia en tanto marca una diferencia epistemológica como un posicionamiento ético-político, ya que no es lo mismo "una actitud cognoscitiva que consiste menos en 'buscar información' sobre su objeto o tema de interés y más en tejer vínculos de interconocimiento con las personas que hacen a ese universo (Quirós, 2014, p. 54).

Ahora bien, ¿cómo reconstruir aquello que se vive, cómo narrar la experiencia, los "mundos vividos"? Y por otra parte, ¿cómo volcarlo en un papel dando cuenta de un modo de conocimiento que es parte de las ciencias sociales? Desde nuestra propuesta, nos centramos en los registros que se convierten en una herramienta por excelencia para poder comunicar los diversos y singulares universos en los cuales nos "insertamos" y en donde proponemos, configuramos y reconfiguramos estrategias de intervención extensionista.

Pero antes de avanzar es necesario detenernos en la pregunta: ¿qué significa "narrar mundos vividos"? Reflexionar en torno a este interrogante nos desafía epistemológicamente, por un lado, para atender a las dimensiones, rastros y rostros que visibilizamos y los que omitimos; por otro lado, ejercitarnos en ello nos permite afinar — desesencializar, descentrar nuestra mirada - para lograr narraciones más fieles a "lo vivido" de aquellos universos.

En tanto, consideramos que el proceso de escribir, narrar experiencias a través de la palabra, el dibujo, la fotografía, entre otros recursos, implica un proceso de descubrimiento y creación. Es en el trabajo artesanal de registrar, de describir, "narrar" y "contar" lo vivido donde radican las preguntas-problemas sobre ese universo en el cual nos relacionamos. Por eso tiene un gran valor metodológico que supone una formación intencionada en proceso donde "reside no solo ni tanto en el producto: describir mundos vívidos para nuestros lectores, como en el propio proceso: por intermedio de la descripción podemos aprender a interrogar y analizar vívidamente el mundo social” (Quirós, 2014, p. 61).

Asimismo, consideramos un desafío valioso el hecho de reconstruir las atmósferas narrativas, es decir, recuperar: ¿qué cosas se dicen? ¿Qué se preguntan y qué se responden? ¿Qué producen esos signos en las situaciones, interacciones, relaciones estudiadas? Y también: ¿cómo se dicen las cosas? ¿Cómo se resuelven? Pensar en la reflexividad de la palabra en acto; la palabra viva nos permite una reflexividad en el mundo social (Quirós, 2014) y es una herramienta de conocimiento más integral y fiel al proceso de la dinámica social propia del acontecer. 


\section{La importancia del registro como parte del proceso de producción de conocimiento en la extensión}

En la construcción de los registros, la escritura toma una centralidad fundamental, como pilar para recuperar lo que acontece, aquello que experimentamos en la interacción con otros y otras así como en la formación académica disciplinar (Castro, 2011). Es por esto que consideramos que los registros habilitan el proceso de producción de conocimientos científicos y la riqueza de un proceso de formación-enseñanza y aprendizaje de nuestros y nuestras estudiantes a partir del valor de la experiencia y fundamentalmente que se encuentren al servicio de la promoción de derechos (Castro, 2011; Vallejos, 2007).

Desde este posicionamiento, consideramos importante desentrañar qué es lo que permite dejar registro de la historia humana en general, pasaporte de sobrevivencia de la cultura. Siguiendo a Sarrot de Budini (2007), podemos decir que " el 'no registro' colabora a la muerte de lo que acontece, al desaprovechamiento de las experiencias, que al no plasmarse en texto, mueren con quien las actuó y vivenció".

Por otra parte, creemos relevante otorgar al registro una dimensión instrumental crítica y emancipadora (Guerra, 2004) en tanto es la primera evidencia en el proceso de intervención e investigación científica que acontece en el ámbito universitario. Esta herramienta se puede convertir en insumo y soporte de la reflexión entre la tensión teoría-práctica, es decir, herramienta indispensable para la mediación entre el sujeto y objeto, entre los sujetos en el proceso de aprendizaje del "saber hacer", en los procesos de comprensión de mundos vividos. El registro forma parte de esa estrategia metodológica que siempre debe hacernos pensar sobre la inconveniencia de la comodidad de aplicar automáticamente la técnica, desvalorizando la creatividad, la novedad y el caos que implica captar los procesos de extensión en territorio.

Ante esto, identificamos diferentes ámbitos y tiempos en los cuales se conjugan y dialogan la teoría y la práctica en el proceso de aprendizaje: por un lado, la vivencia y experiencia del encuentro con otra u otro en "el terreno" donde registramos, describimos, recolectamos eso que pasa y también lo que nos pasa, atraviesa y modifica. Por otro lado, un ámbito de reflexión donde realizamos un "descentramiento teórico" en la relectura de aquellos registros que nos permiten establecer asociaciones que estaban invisibles, formulamos preguntas impensadas, generamos hipótesis.

Esta tarea es posible si el registro existe, si podemos documentar aquella experiencia para que en el proceso de aprehender nos vaya allanando el camino y tengamos la oportunidad de nuevos descubrimientos en esta labor que llamamos "artesanía intelectual" (Wrigth Mills, 2009). Asimismo, la interpretación, el proceso de reflexión y análisis, es lo que permite alejarse de la impronta "normativa" de la disciplina y habilitar la posibilidad de crear estructuras argumentativas novedosas, corpus conceptuales más acordes a las circunstancias del contexto de la vida social en su complejidad.

Pero el registro como herramienta no es neutral (Bourdieu, 1994) sino que conlleva una intencionalidad y direccionalidad, forma parte de una estrategia metodológica. Es muy importante que podamos hacer consciente este aspecto ya que nos permite "afinar nuestro ojo" para enriquecer nuestros registros y por ende, nuestros análisis. Por eso consideramos que es una herramienta de poder, es decir, presenta un carácter eminentemente político en cuanto a visibilizar o invisibilizar prácticas, experiencias, actores, tramas sociales y todo 
aquello que acontece. Puede ser una herramienta de control o de transformación según la finalidad que se le otorgue. $Y$ esto se encuentra vinculado con la dimensión ética, la cual consideramos parte imprescindible en las estrategias de registros que se establecen según las posibilidades y limitaciones del contexto en el que se lleva a cabo el proyecto. Por último, queremos recuperar un aspecto fundamental que Bourdieu denomina "vigilancia epistemológica" para no incurrir en trasgresiones que atenten contra la privacidad y el resguardo de la identidad de todos los actores y actoras, instituciones y organizaciones partícipes del proceso. El registro cumple una función social de inscribir "sujetos de conocimientos y acción", por lo tanto hacerlo hablar implica realizarlo dentro de los recortes que el objeto construido nos habilita y los acuerdos que se van estableciendo en la trama de vínculos que se construye a lo largo del proceso.

\section{El valor pedagógico del registro en el proceso de formación académica para la extensión}

Ya hemos establecido que el registro es una técnica central en el desarrollo de estrategias de intervención pedagógico-social para propiciar la comprensión e indagación de la acción que pretende captar, describir, analizar e interpretar, y lograr reflexiones superadoras. Su realización ha de posibilitar analizar densamente el mundo de las prácticas experienciales en las que confluyen múltiples sujetos, escenarios, posicionamientos ético-políticos desde la dinámica de las cátedras y proyectos académicos vigentes en la universidad hoy.

Desde nuestro posicionamiento, consideramos necesario que los registros habiliten la posibilidad de construir mirada, interpretar y recrear los procesos propios y grupales, aportando a la constitución de espacios deliberativos que estimulen la identificación de aciertos y errores, de desigualdades en las percepciones y vivencias, lo que potenciará la imaginación para nutrir y redireccionar perspectivas y acciones teórico-prácticas.

En este sentido, creemos que los procesos de Prácticas de Extensión de Educación Experiencial (PEEE) y los Proyectos de Extensión de Interés Social (PEIS) requieren de la construcción de relatos por diversas razones. El relato tiene la pretensión de un conocimiento que compartimos de forma oral o escrita y brinda el detalle respecto de los procesos extensionistas en los que nos encontramos, pudiendo ser en ellos protagonistas activos (cuando nos involucramos participando del proceso de decisiones y como actores), o pasivos (cuando asumimos posiciones de carácter evasivas, a veces disociadas de los propósitos perseguidos, o solo tendientes a cumplir con la formalidad del proyecto). Por otra parte, la relectura de esos relatos permite formular preguntas, establecer relaciones, encontrar conceptos que en la experiencia vivenciada no hubieran sido factibles ya que necesitamos un tiempo para procesar la información, avanzar en el análisis y producir síntesis.

El registro es un tipo de práctica discursiva que construye realidad, saber y poder. Puede convertirse en un auténtico instrumento de poder al visibilizar o invisibilizar prácticas, experiencias, protagonismos, actorías, conocimientos, vínculos y tramas sociales. Así, su finalidad va a estar relacionada directamente con la intencionalidad que se haya trazado para el proyecto en el que estemos involucrados y con sus objetivos. Esto no tiene que limitar o suprimir su impronta creativa, abierta al reconocimiento de la novedad e incertidumbre propias de los procesos sociales. Tienen que posibilitar la interpelación de los propios conoci- 
mientos a partir de las palabras del relato producido, la propia voz y sentimientos, y la de los actores que no pueden quedar por fuera del registro si este aspira a ser un relato pertinente y significativo en el marco de los proyectos "sociales".

Los registros son reconstrucciones dinámicas y personales de las experiencias donde los y las estudiantes dan significado a lo que vivenciaron mediante un proceso reflexivo que la misma escritura les permite en el momento de la construcción. Estas narrativas estructuran las prácticas y el lenguaje de las mismas, visibilizan los propósitos de esas prácticas, los imprevistos, las transformaciones, como también son insumos de relectura para constituirse y colaborar en otras producciones de conocimiento. Es por esto que las narraciones que los y las estudiantes y el equipo extensionista en su conjunto construyen a partir de sus registros en cuanto a las observaciones y experiencias que vivencian en el territorio requieren de estrategias pedagógicas curriculares que promuevan el ejercicio del "Pensamiento Epistémico" (Zemelman, 2001). Con esta premisa desafiante, consideramos sumamente enriquecedor hacer visible la importancia del registro como instrumento pedagógico. El proceso de aprendizaje y de enseñanza que apuesta a la relación dialógica entre docente y estudiantes a través de la socialización de producciones escritas, intercambios de ideas, debates, conlleva a desandar un proceso de formación profesional que propicia que el "pensamiento se pueda colocar ante las circunstancias... construyendo una relación de conocimiento sin que esta quede encerrada en un conjunto de atributos" (Zemelman, 2001, p. 3).

Desde esta perspectiva, permiten socialmente objetivar, legitimar y difundir experiencias y prácticas para ponerlas en deliberación y circulación pública, además de evaluar en proceso los proyectos e ir redireccionándolos con el aporte colectivo al hacerse factible su exposición. Por eso es preciso que los proyectos contemplen un espacio de reflexión que "permite optimizar los procesos de apropiación activa y crítica de los contenidos curriculares en tensión con las experiencias provenientes de la inserción institucional a través de las prácticas preprofesionales" (Castro, 2011).

A modo propositivo, queremos resaltar la centralidad de generar espacios de taller en el marco de los proyectos como dispositivos que contemplen la conformación de pequeños grupos donde se habilite la posibilidad de intercambio y confrontación de ideas, la recuperación de lo registrado y mediaciones teóricas que dejen abierta la puerta a la emergencia de lo nuevo. Abrirse a la diversidad, diferencia y desigualdad tanto en el campo como en la escritura nos da una valiosa oportunidad para asumir la incomodidad de involucrarnos en el mundo social, reconociendo su rostro contradictorio y controversial, que siempre desafía aquello que se supone políticamente correcto y lo encubre dejando intersticios para que lo dicho, lo nombrado y reconocido, no sean suficientes. Esto implica renovar las preguntas acerca de: ¿para qué sirve el conocimiento académico? ¿Para quiénes? Reflexionar sobre el sentido político de nuestras acciones, producciones de conocimiento y aportes conceptuales.

Las experiencias que habilitan y habitan los proyectos de extensión tienen un importante valo en tanto son realidades vividas más que una categoría de análisis. En los encuentros de sujetos de experiencia multidimensional, que se construyen desde sus identidades socioculturales múltiples basadas en etnicidad, género, religión, sexualidad, clase, color, clan, nacionalidad, así como por sus deseos, sueños, expectativas, en un entramado complejo, singular, situado y cambiante. Los registros recuperan aspectos que constituyen las 
propias vidas, vidas que hacen a la dimensión humana del mundo social, a su rostro humano en clave académica de humanidad.

\section{Referencias bibliográficas}

Argumedo, A. (1993). Los silencios y las voces en América Latina. Ediciones del Pensamiento Nacional Camilloni, A. (2013). La inclusión de la educación experiencial en el currículum universitario. En Menéndez, G. (Comp.), Integración docencia y extensión. Otra forma de aprender y de enseñar. Ediciones UNL.

Castro, S. (2011). El registro en la intervención: una reflexión epistemológica. En Arias, A.; García Godoy, B. y Manes, R. (Comps.), Trabajo social, lecturas teóricas y perspectivas: aportes para repensar la formación profesional desde la intervención. Universidad de Buenos Aires.

Cifuentes Gil, R. M. (2010). Participación en foro sobre lecciones aprendidas en torno a la Sistematización. RELALC.

Freire, P. (1996). Pedagogía de la esperanza. Siglo XXI Editores.

Goldman, M. (2006). Alteridade e experiência: antropologia e teoria etnográfica. Etnográfica, 10(1), 161-173.

Guber, R. (2001). La etnografía. Método, campo y reflexividad. Norma.

(2004). El salvaje metropolitano. Paidós.

Guerra, Y. (2004). Instrumentalidad del proceso de trabajo y servicio social. XVIII Seminario Latinoamericano de Escuelas de Trabajo Social. La cuestión Social y la formación profesional en Trabajo Social en el contexto de las nuevas relaciones de poder y la diversidad latinoamericana. San José, Costa Rica.

Larrosa, J. (2009). Sobre la experiencia. http://files.practicasdesubjetivacion.webnode.es/2000000189863d9a585/_la_experiencia_Larrosa.pdf

Menéndez, G. (2011). Los desafíos presentes y futuros de la extensión universitaria. +E: Revista de Extensión Universitaria, 1(1), 22-31. https://doi.org/10.14409/extension.v1i1.443

Menéndez, G. (Comp.) (2013). Integración docencia y extensión. Otra forma de aprender y de enseñar. Ediciones UNL.

Quirós, J. (2014). Etnografiar mundos vividos. Desafíos del trabajo de campo, escritura y enseñanza. Publicar en Antropología y Ciencias Sociales, (17), 47-65.

Rafaghelli, M. (2013). La dimensión pedagógica de la extensión. En Menéndez, G. (Comp.), Integración docencia y extensión. Otra forma de aprender y de enseñar. Ediciones UNL. http://biblioteca.clacso.edu.ar/ Argentina/fcjs-unl/20171101043348/pdf_1172.pdf

Rockwell, E. (2005). Del campo al texto. Reflexiones sobre el trabajo etnográfico. Conferencia en Sesión Plenaria del Primer Congreso de Etnología y Educación. Universidad Castilla-La Mancha. https://cursoensenada2011.files.wordpress.com/2011/05/rockwell-del-campo-al-texto-2005.pdf

Sarrot de Budini, E. (2007). Registro y no registro. En Cuadernillo Cursos de Profundización "El informe/ el registro" (pp. 47-58). Área de Capacitación y Posgrado. Sec. de Extensión e Investigación. FTS-UNER. http:// www.fts.uner.edu.ar/publicaciones/publicaciones/cuadernillos/texto/el\%20informe_el\%20registro.pdf

Vallejos, I. (2007). El registro de campo: su utilización en los procesos de aprendizaje de la intervención profesional de Trabajo Social. En Cuadernillo Cursos de Profundización "El informe/ el registro" (pp. 59-65). Área de Capacitación y Posgrado. Sec. de Extensión e Investigación. FTS-UNER. http://www.fts.uner.edu.ar/publicaciones/publicaciones/cuadernillos/texto/el\%20informe_el\%20registro.pdf

Wrigth Mills, C. (2009). Sobre Artesanía Intelectual. Trabajo y Sociedad. Indagaciones sobre el trabajo, la cultura y las prácticas políticas en sociedades segmentadas, 13(XII). https://www.unse.edu.ar/trabajoysociedad/13_WRIGHT_MILLS_ARTESANIA_INTELECTUAL.pdf 
Zapata, L. \& Genovesi, M. (2013). Jeanne Favret- Saada: ser afectado como medio de conocimiento en el trabajo de campo antropológico. Avá, (23), 49-67.

Zemelman, H. (2001). Pensar teórico y pensar epistémico: los retos de las ciencias sociales latinoamericanas. Transcripción de la conferencia magistral dictada por el Dr. Zemelman en la Universidad de la Ciudad de México. (Texto editado revisado por el Dr. Zemelman.) http://www.ipecal.edu.mx/Biblioteca/Documentos/ Documento7.pdf 